\title{
Little boxes: A simple implementation of the Greenberger, Horne, and Zeilinger result for spatial degrees of freedom
}

\author{
John D. Norton \\ Department of History and Philosophy of Science and Center for Philosophy of Science, \\ University of Pittsburgh, Pittsburgh, Pennsylvania 15260
}

(Received 12 May 2010; accepted 14 September 2010)

A Greenberger, Horne, and Zeilinger-type construction is realized in the position properties of three
particles whose wavefunctions are distributed over three two-chambered boxes. The same system
is modeled more realistically using three spatially separated, singly ionized hydrogen molecules. (C) 2011 American Association of Physics Teachers.

[DOI: $10.1119 / 1.3531943$ ]

\section{INTRODUCTION}

In a celebrated critique, Einstein used entangled systems in an attempt to show that the quantum state gives an incomplete description of the properties possessed by a quantum system. ${ }^{1}$ In a celebrated rejoinder, Bell, ${ }^{2}$ and those who developed his approach, showed that no theory employing hidden states that are spatially separate and local could recover the same predictions as quantum theory.

Most workers have developed Bell's ideas in terms of the internal degrees of freedom associated with spin because spins are simpler theoretically and lend themselves to experimental tests. ${ }^{3}$ These analyses preclude hidden spin properties. What of the spatial degrees of freedom discussed early in the development of quantum theory that are associated with the spread of the wavefunction?

This paper will show how the same analysis can be applied directly to spatial degrees of freedom and that they preclude hidden position properties. In so far as possible, the analysis will employ only the ideas that a quantum system can be represented by a wavefunction and that measurement collapses the wavefunction. In particular, the analysis will adapt Bernstein's ${ }^{4}$ simplified analysis of a Greenberger, Horne, and Zeilinger (GHZ)-type construction to spatial degrees of freedom.

The spatial degrees of freedom will be introduced through an example that Einstein communicated to Schrödinger shortly after the well known Einstein, Podolsky, and Rosen (EPR) paper was written. ${ }^{1}$ While discussing the essential ideas of the EPR paper, Einstein illustrated the notion of incompleteness with the idea of a ball that will assuredly appear in one of two boxes. Because the ball's wavefunction is spread over the two boxes, Einstein suggested that the wavefunction provides an incomplete description of the ball. Einstein's account will be given in Sec. II. An example, a two-chambered box with a particle, will be described in Sec. III. The division into two chambers will give the added spatial degrees of freedom used to implement the GHZ construction. A realistic implementation of the system is found in molecular orbital chemistry through the singly ionized hydrogen molecule, $\mathrm{H}_{2}{ }^{+}$. This implementation precludes the concern that the highly idealized system of two-chambered boxes might introduce spurious idealizations that are responsible for the effects.

Einstein's attempt to show incompleteness depends on the properties of entangled systems. These properties will be described briefly in Sec. IV. The EPR analysis, sketched in Sec. $\mathrm{V}$, uses measurements of the properties of one system to discern the properties possessed by another spatially distant system that is entangled with it. Section VI describes a system of three entangled particles that are spread over three two-chambered boxes. The hidden position property sought by Einstein's methods is whether each of the boxes' particles is in the left or the right chamber.

In Sec. VII, we shall see how possible measurements on any two of the boxes enable the remote determination of the position properties of the third. The totality of such measurements constrains the position properties that must be possessed by the particles. Two sorts of measurements will be described. One is the straightforward position measurement of which chamber hosts the particle. The other is the determination of whether the particle's wavefunction is spread over the two chambers in a "bonding" or "antibonding" state, drawing on a notion from molecular orbital theory. The two sets of measurements lead to contradictory ascriptions of the hidden position properties, which establishes the failure of Einstein's attempt to show the incompleteness of quantum theory.

\section{EINSTEIN'S BOXES}

The EPR paper was received by Physical Review on March 25, 1935 and published on May 15, 1935. In a letter of June 19, 1935, Einstein expressed his dissatisfaction with the exposition. "For reasons of language," Einstein explained to Schrödinger, "[it] was written by Podolosky after many discussions. But still it has not come out as well as I really wanted; on the contrary, the main point was, so to speak, buried by the erudition." ${ }^{, 6} \mathrm{He}$ then identified the real problem as residing in the meaning of the assertions that a description of reality is complete or incomplete. To explain the difference, Einstein introduced the following illustration. ${ }^{?}$

“... I want to explain the [se assertions] through the following illustration: In front of me are two boxes with hinged lids, into which I can see when the lids are opened; the latter is called 'making an observation. There is also a ball there that will always be found in one or other of the boxes, if one makes an observation."

Einstein then distinguished his view of the incompleteness of quantum description ("no") from the standard view of completeness ("yes"): 


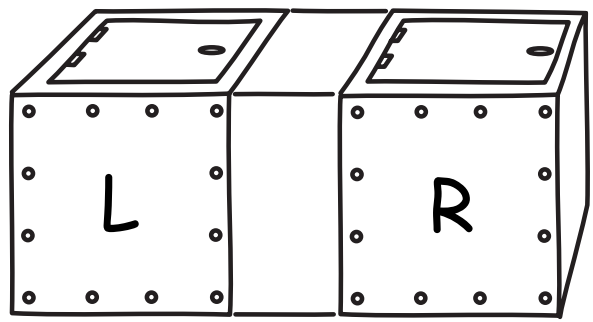

Fig. 1. A two-chambered box.

"I now describe a state as follows: The probability that the ball is in the first box is 1/2.-Is this a complete description?

No: A complete statement is the ball is in the first box (or is not). That, therefore, is how the characterization of the state must appear in a complete description.

Yes. Before I open the box, the ball is by no means in one of the two boxes. Being in a definite box only comes about when I lift the lid...."

\section{A PARTICLE CONFINED TO A TWO- CHAMBERED BOX}

In the following, we will base the analysis on Einstein's example of a ball that is spread over two boxes. To keep the analysis tractable, Einstein's two boxes will be joined together as two spatially separated compartments or chambers L (left) and R (right) of one larger box, as shown in Fig. 1. Einstein's ball will be a single particle.

The simplest quantum mechanical implementation of this system is a single particle confined by two infinitely deep square potential wells. ${ }^{8}$ Figure 2 shows one of many possible wavefunctions.

The two quantum wavefunctions $\Psi_{\mathrm{L}}$ and $\Psi_{\mathrm{R}}$ are normalized solutions of the Schrödinger equation for each potential well individually. The combined wavefunction is their normalized sum

$$
\Psi_{\beta}=(1 / \sqrt{2})\left(\Psi_{\mathrm{L}}+\beta \Psi_{\mathrm{R}}\right),
$$

where $\beta$ is a relative internal phase factor of unit norm, such as $1,-1, \mathrm{i},-\mathrm{i}$.

A more realistic implementation of the system is a singly ionized hydrogen molecule, $\mathrm{H}_{2}{ }^{+}$. This molecule consists of two protons, whose positive charges create a potential well

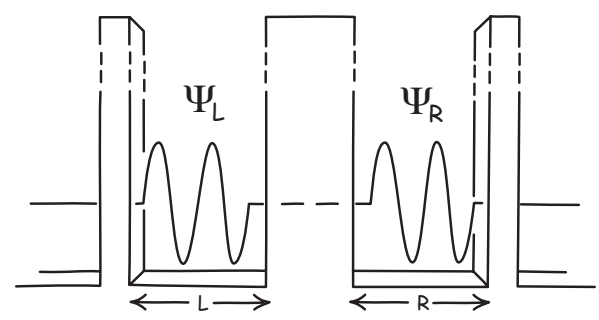

Fig. 2. Particle in two infinitely deep square potential wells.

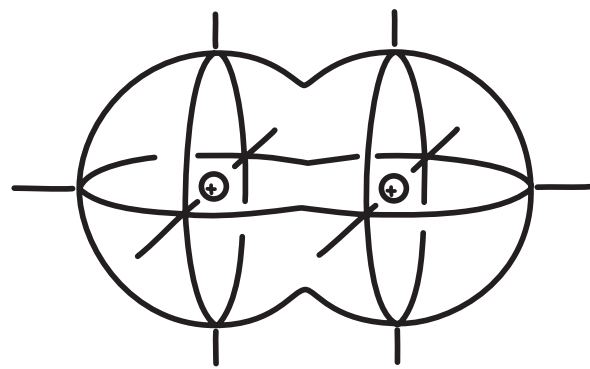

Fig. 3. Singly ionized hydrogen molecule, $\mathrm{H}_{2}^{+}$.

with two chambers centered on each proton. The single electron of the ionized molecule is spread across these two chambers. In the standard linear combination of atomic orbital (LCAO) approximation of molecular orbital theory, ${ }^{9}$ the molecule is approximated by a linear superposition of two $1 \mathrm{~s}$ electron orbitals of the ordinary hydrogen atom (see Fig. 3).

The bond that joins the two atoms results from the overlapping of these two orbitals. It will be important for the following analysis that the compartments be disjoint spatially. Therefore, the relevant system consists of an $\mathrm{H}_{2}{ }^{+}$molecule in which the two nuclei have been separated sufficiently in space so that the two $1 s$ orbitals no longer overlap, as shown in Fig. 4.

Overlapping is avoided almost completely by separating the two nuclei by about $10 \mathrm{bohr}$ radii. ${ }^{10}$ If the two $1 \mathrm{~s}$ orbitals of the separated $\mathrm{H}_{2}^{+}$molecule are $\Psi_{\mathrm{L}}$ and $\Psi_{\mathrm{R}}$, then the wavefunction of the molecule's single electron is given by Eq. (1).

Almost all of the details of the particle wavefunction in a two-chambered box are immaterial to the following analysis. All that is important is that there is a particle whose wavefunction is nonvanishing only in two disjoint regions of spaces $L$ and $R$. The wavefunctions of the particle, if it were confined to each region individually, are $\Psi_{\mathrm{L}}$ and $\Psi_{\mathrm{R}}$. Many possible wavefunctions are admissible for $\Psi_{\mathrm{L}}$ and $\Psi_{\mathrm{R}}$. For a potential well, they correspond to the different energy eigenstates of a particle trapped in such a potential, and for a separated $\mathrm{H}_{2}^{+}$they correspond to the various orbitals of a hydrogen atom. All that matters is that we fix on a particular pair of wavefunctions $\Psi_{\mathrm{L}}$ and $\Psi_{\mathrm{R}}$ and use them for the remainder of the analysis.

\section{ENTANGLEMENT AND THE MEASUREMENT OF DISTANT PARTICLE STATES}

The EPR analysis depends essentially on the entanglement of systems. The way entanglement of spatial degrees of freedom affects quantum measurement is readily recovered from examining the spatial collapse of the wavepacket under a measurement.

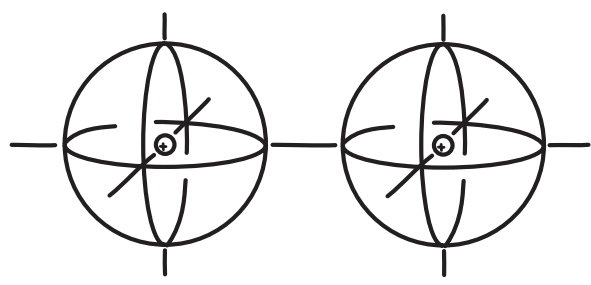

Fig. 4. Separated singly ionized $\mathrm{H}_{2}{ }^{+}$molecule. 

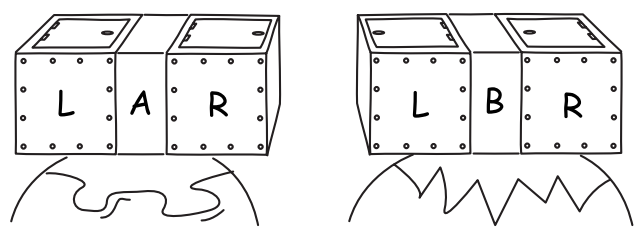

Fig. 5. Two two-chambered boxes.

The simplest case arises when we have two twochambered boxes, A and B (see Fig. 5), which are widely separated in space. The particles of each of these boxes may be entangled in different ways. One simple way is that the presence of A in its L chamber is associated with B's presence in its $\mathrm{L}$ chamber, and similarly for the $\mathrm{R}$ chambers. The wavefunction of the resulting entangled state is

$$
\Psi_{\mathrm{AB}}=(1 / \sqrt{2})\left(\Psi_{\mathrm{A}, \mathrm{L}} \Psi_{\mathrm{B}, \mathrm{L}}+\Psi_{\mathrm{A}, \mathrm{R}} \Psi_{\mathrm{B}, \mathrm{R}}\right) .
$$

Here, $\Psi_{\mathrm{A}, \mathrm{L}}$ and $\Psi_{\mathrm{A}, \mathrm{R}}$ are wavefunctions in the position space of particle $\mathrm{A}$, and $\Psi_{\mathrm{B}, \mathrm{L}}$ and $\Psi_{\mathrm{B}, \mathrm{R}}$ are wavefunctions in the position space of particle B. $\Psi_{\mathrm{AB}}$ is not a wavefunction in a three-dimensional space, but is a wavefunction in the sixdimensional configuration space formed by the product of the two spaces of particles A and B.

The wavefunction $\Psi_{\mathrm{AB}}$ is shown in Fig. 6 in its configuration space; one spatial dimension for each of the $\mathrm{A}$ and $\mathrm{B}$ particles is shown and the remaining four dimensions are suppressed. The wavefunction is nonzero only in two regions of configuration space. One coincides with the $\mathrm{L}$ regions of both $\mathrm{A}$ and $\mathrm{B}$ spaces, LL, and corresponds to the product $\Psi_{\mathrm{A}, \mathrm{L}} \Psi_{\mathrm{B}, \mathrm{L}}$ of Eq. (2). The other coincides with the $\mathrm{R}$ regions of both A and B spaces, RR, and corresponds to the product $\Psi_{\mathrm{A}, \mathrm{R}} \Psi_{\mathrm{B}, \mathrm{R}}$ of Eq. (2).

Suppose that we perform a measurement on particle $A$ that determines whether the particle is in the $\mathrm{L}$ or the $\mathrm{R}$ chamber. In the standard interpretation of quantum theory, the effect of this measurement is to collapse the wavefunction to one that is nonzero only in one of the $\mathrm{L}$ or $\mathrm{R}$ regions of A's space. That is, the measurement will collapse $\Psi_{\mathrm{AB}}$ to one of the regions LL or RR of the configuration space. If the outcome of the measurement on the A particle is $\mathrm{L}$, then the wavefunction would collapse to the LL region. This collapse

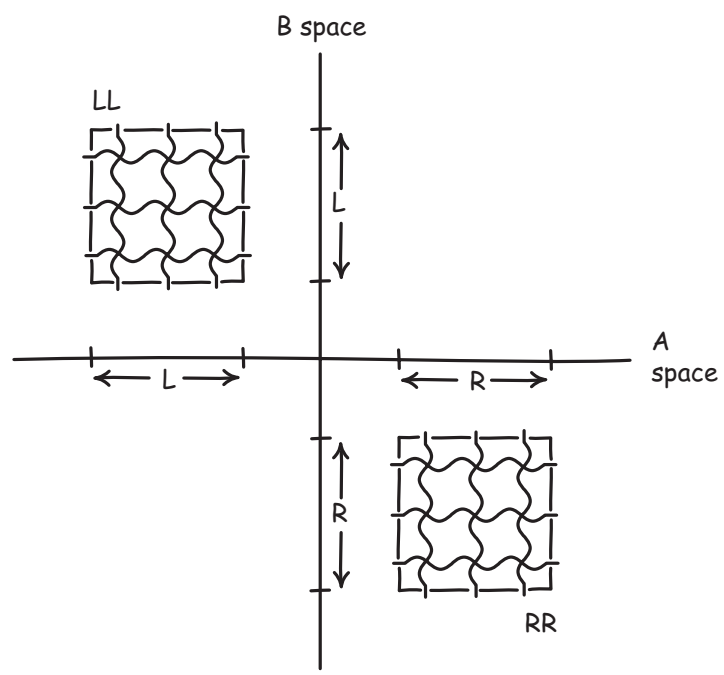

Fig. 6. Configuration space of two correlated particles of boxes A and B. would alter the wavefunction of $B$, which would now only be nonzero in its L region. That is, the wavefunction of Eq. (2) has collapsed to just one of its terms

$$
\Psi_{\mathrm{AB}}=\Psi_{\mathrm{A}, \mathrm{L}} \Psi_{\mathrm{B}, \mathrm{L}} \text {. }
$$

As a result, we can now be certain that any subsequent measurement of the position of the B particle will reveal it to be in its $\mathrm{L}$ region.

This correlation among measurement outcomes is the important fact about entanglement for our purposes. Even though we have measured the A particle only, we are now certain about the outcome of measurements were they to be performed on the B particle, light years away.

\section{THE EPR ANALYSIS}

Einstein's objection to quantum theory was that its standard interpretation was incomplete. That is, specifying the wavefunction associated with a particle does not fix all the physical properties of the particle. If the particle's wavefunction is spread over the two chambers of the box, then standard quantum theory tells us that the particle is neither properly in one or other of the chambers. Einstein suggested that the particle has further factual properties, not captured in the wavefunction, which correspond to its localization in one or other of the chambers.

Einstein's strategy for establishing this incompleteness was first advanced in the EPR paper and then by himself in some of Einstein's later publications. ${ }^{1}$ The analysis depended on the behavior of entangled systems under measurement, as sketched in Sec. IV. Measurement of the position of A enables us to know what a position measurement of B would yield, even though B is so remote from A that the physical process of our measurement of A could have no effect on $B$. This fact establishes that the B particle had a definite position property all along, even though the original wavefunction did not express it.

This analysis depends on an inference from our being able to predict assuredly the outcome of a measurement on the B particle to the possession by the B particle of a property. The assumption that justified that inference is of central importance to the EPR analysis and stated in italics on the first page of the EPR paper, "If, without in any way disturbing a system, we can predict with certainty (i.e. with probability equal to unity) the value of a physical quantity, then there exists an element of physical reality corresponding to this physical quantity." We will refer to this assumption as the "EPR reality criterion."

There is an important counterfactual element in the EPR analysis. ${ }^{11}$ It does not require that we perform the measurements on the A particle to infer the possession of position properties by the B particle. All that matters is that we know what would happen if we were to perform the measurement, even when, counter to the facts, we do not perform it. If we could predict assuredly that there would be some definite position measured on the remote B particle, then we could infer that the remote particle possesses some definite position property. All we lose by not performing a measurement on the A particle is that we do not know which position property is possessed by the remote $\mathrm{B}$ particle. Because we need not actually perform the measurements, analogous reflections on the possibility of measurements on the B particle lead to the corresponding conclusion for the A particle: It too possesses a definite position property not expressed completely by its 

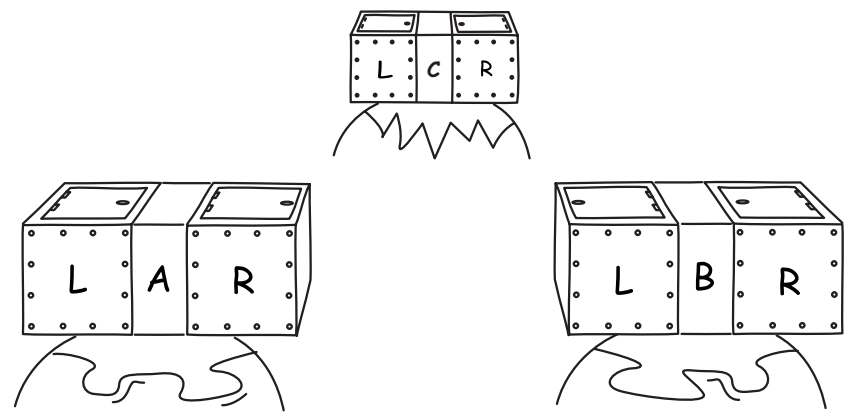

Fig. 7. Three two-chambered boxes.

wavefunction. The counterfactual aspect is essential if we are to infer that both $\mathrm{A}$ and $\mathrm{B}$ particles possess more position properties than expressed by their wavefunctions. Because we can perform the measurement on at most one of the two entangled particles, then under the standard theory, measurement on one alters the combined system. ${ }^{12}$

Howard ${ }^{5}$ traced the presence of two important assumptions. First is separability: The assumption that the two spatially remote systems have independent existences. Second is locality: The assumption that effects between the systems propagate at luminal speeds or less. The first assumption affirms that the remote particle has properties independent of the local particle being measured, and the second assures us that whatever disturbances are introduced by measurement will initially affect only the local particle.

\section{THREE TWO-CHAMBERED BOXES, ENTANGLED}

The failure of the EPR attempt to show the incompleteness of quantum theory was demonstrated by Bell ${ }^{2}$ and others. The failure is demonstrated by showing that no physical theory, constrained by separability and locality, can reproduce the empirical predictions of quantum theory. Most analyses follow Bell in considering two entangled systems, such as two spin half particles in a singlet state. In that case, the empirical failure of separable local theories is their failure to reproduce the correlations between measurement outcomes predicted by quantum theory.

A significant conceptual simplification was achieved by Greenberger, Horne, and Zeilinger. ${ }^{13}$ If we consider just two entangled systems, the demonstration of the failure of the EPR analysis works indirectly by showing that a local separable theory cannot return the statistical correlations among measurements predicted by quantum theory. In the GHZ approach, more than two entangled systems are considered. This approach enables the EPR reality criterion to be applied directly. From it and the predictions of quantum theory, we can infer the properties a spatially remote system must possess and note that the ensuing requirements are contradictory.

The entangled system to be developed here is a version of a GHZ system. It is the one described in Ref. 14 and simplified by Bernstein. ${ }^{4}$ The analysis has been modified by substituting spatial degrees of freedom for the spin degrees of freedom. ${ }^{15}$ The system consists of three spatially remote twochambered boxes, A, B, and C, with one particle in each box, as shown in Fig. 7.

To display the failure of the EPR analysis, the entanglement of the particles in the three boxes is a little more com- plicated than that of the two particle system of Eq. (2). Each particle is spread over the two chambers of its box. For example, there are two ways the A particle is spread:

$$
\begin{aligned}
& \Psi_{\mathrm{A},+i}=(1 / \sqrt{2})\left(\Psi_{\mathrm{A}, \mathrm{L}}+i \Psi_{\mathrm{A}, \mathrm{R}}\right), \\
& \Psi_{\mathrm{A},-i}=(1 / \sqrt{2})\left(\Psi_{\mathrm{A}, \mathrm{L}}-i \Psi_{\mathrm{A}, \mathrm{R}}\right) .
\end{aligned}
$$

Equation (4a) has a relative internal phase factor $\beta$ of $+i$, and Eq. (4b) has a relative internal phase factor of $-i$. Wavefunctions for the $\mathrm{B}$ and $\mathrm{C}$ boxes are defined analogously. The three particles are entangled in the state

$$
\Psi_{\mathrm{ABC}}=(1 / \sqrt{2})\left(\Psi_{\mathrm{A},+i} \Psi_{\mathrm{B},+i} \Psi_{\mathrm{C},+i}-\Psi_{\mathrm{A},-i} \Psi_{\mathrm{B},-i} \Psi_{\mathrm{C},-i}\right) .
$$

In the remainder of the paper, we will apply the EPR reality criterion to this state to determine the properties this criterion attributes to it (its "elements of physical reality") and then show that the resulting requirements are self-contradictory.

\section{MEASUREMENT OF POSSESSED PROPERTIES}

We shall ascertain the position properties of the three particles of the entangled state $\Psi_{\mathrm{ABC}}$ by considering the outcomes of two sorts of measurements: Position measurements and "bonding/antibonding" measurements. These measurements on two of the particles will enable us to predict the outcome of a position measurement on the third remote particle. Using the EPR reality criterion, we shall thereby learn a property possessed by the remote particle. The totality of all possible measurements will place strong restrictions on the position properties that may be possessed by the three particles. It will turn out that the two different sorts of measurement lead to contradictory restrictions.

\section{A. First procedure: Position measurement}

The form in which $\Psi_{\mathrm{ABC}}$ is written in Eq. (5) is not conducive to reading off these predictions. We can obtain an equivalent expression for $\Psi_{\mathrm{ABC}}$ if we substitute for each term in Eq. (5) expressions for $\Psi_{\mathrm{A},+i}, \Psi_{\mathrm{A},-i}, \Psi_{\mathrm{B},+i}, \ldots$ from Eq. (4). Multiplying out the terms leads to an unwieldy expression with 16 terms, most of which cancel, leaving

$$
\begin{aligned}
\Psi_{\mathrm{ABC}}= & (i / 2)\left(\Psi_{\mathrm{A}, \mathrm{L}} \Psi_{\mathrm{B}, \mathrm{L}} \Psi_{\mathrm{C}, \mathrm{R}}+\Psi_{\mathrm{A}, \mathrm{L}} \Psi_{\mathrm{B}, \mathrm{R}} \Psi_{\mathrm{C}, \mathrm{L}}\right. \\
& \left.+\Psi_{\mathrm{A}, \mathrm{R}} \Psi_{\mathrm{B}, \mathrm{L}} \Psi_{\mathrm{C}, \mathrm{L}}-\Psi_{\mathrm{A}, \mathrm{R}} \Psi_{\mathrm{B}, \mathrm{R}} \Psi_{\mathrm{C}, \mathrm{R}}\right) .
\end{aligned}
$$

We can read off from Eq. (6) how position measurements on two particles enable us to predict the outcome of a position measurement on the other particle. For example, if position measurements of the A and B particles both yield L, then the state collapses to the first term

$$
\Psi_{\mathrm{ABC}}=\Psi_{\mathrm{A}, \mathrm{L}} \Psi_{\mathrm{B}, \mathrm{L}} \Psi_{\mathrm{C}, \mathrm{R}},
$$

and we know that the $\mathrm{C}$ particle must return the position $\mathrm{R}$ on measurement. These four terms correspond to four regions in the configuration space of particles A, B, and C, as shown in Fig. 8. The measurement described in Eq. (7) would collapse the wavefunction to the one marked LLR.

A measurement of the positions of any two particles will collapse the wavefunction to one of the four regions LLR, LRL, RLL, and RRR, where LLR indicates A position L, B position $\mathrm{L}, \mathrm{C}$ position $\mathrm{R}$, etc. We read the following rule from these four possibilities: 


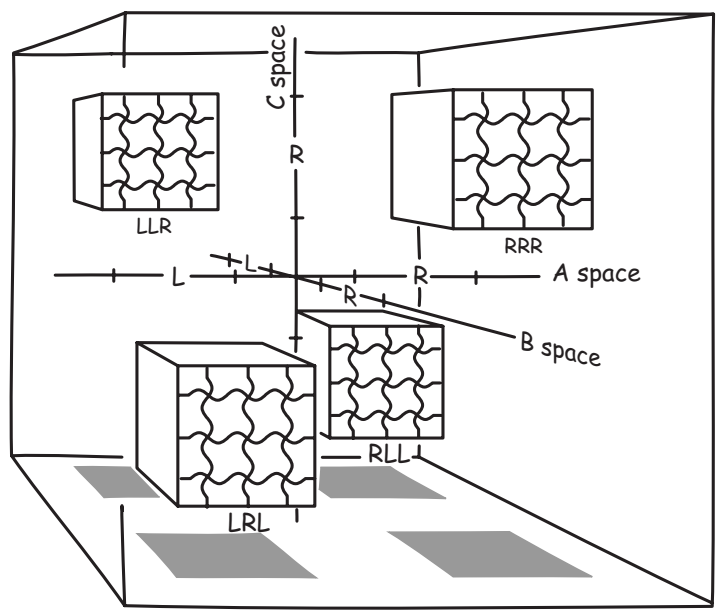

Fig. 8. Configuration space of the three entangled particles of $\Psi_{\mathrm{ABC}}$.

Rule: Same - R, Different - L.

If we measure two particles to have the same position, then the remaining particle will return position $\mathrm{R}$ on measurement. If we measure two particles to have different positions, then the remaining particle will return position $\mathrm{L}$ on measurement. Here, "same" means both L or both R, and "different" means one L and one R.

\section{B. Applying the EPR reality criterion}

We now apply the EPR reality criterion to these results that, so far, only concern measurement. When the rule predicts assuredly which position property would be measured for a remote particle, we infer that the particle possesses it as a hidden position property.

We could choose to measure the particle positions of any pair of systems: The pair $\mathrm{BC}$, the pair $\mathrm{AC}$, or the pair $\mathrm{AB}$. Then, the rule ${ }^{16}$ in Eq. (8) would enable to us predict with certainty what we would measure for the position of the particle of the third system. Recall that the EPR analysis allows us to assemble conclusions that are derived from considering many different measurements that, counterfactually, are not performed. Hence, we can pool all these outcomes and conclude that each of the $\mathrm{A}, \mathrm{B}$, and $\mathrm{C}$ particles really possess the property of $\mathrm{L} / \mathrm{R}$ position.

If we consider the possible position measurements of the three possible pairings of the particle, we may ask if the members of each pair are the same or different. There are only two cases: (a) All three pairs have the same position. (b) One pair has the same position and the remaining two pairs have different positions. The position properties of these two cases must conform to the rule in Eq. (8) Applying it to case (a) tells us that all three position properties must all be $\mathrm{R}$. Applying the rule to case (b) tells us that two position properties are L and one is R. That is, there are only four possible distributions of position properties

LLR, LRL, RLL, and RRR.

\section{Second procedure: Bonding/antibonding measurement}

A position measurement determines whether a particle in the two-chambered box is in the left or right chamber, that is, whether the particle manifests itself as $\Psi_{\mathrm{L}}$ or $\Psi_{\mathrm{R}}$ on mea-

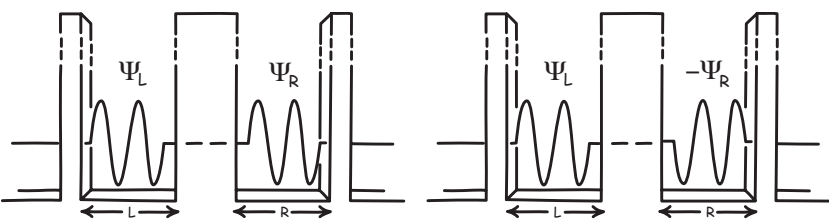

Fig. 9. $\Psi_{+1}$ and $\Psi_{-1}$ for two square wave potential wells.

surement. A second measurement determines whether the particle manifests as $\Psi_{+1}$ or $\Psi_{-1}$, that is, as the normalized sum or difference of $\Psi_{\mathrm{L}}$ and $\Psi_{\mathrm{R}}$,

$$
\Psi_{+1}=(1 / \sqrt{2})\left(\Psi_{\mathrm{L}}+\Psi_{\mathrm{R}}\right), \quad \Psi_{-1}=(1 / \sqrt{2})\left(\Psi_{\mathrm{L}}-\Psi_{\mathrm{R}}\right) .
$$

The wavefunctions corresponding to the two cases are shown in Fig. 9 for a particle spread over two square wave potential wells. $\Psi_{+1}$ is on the left and $\Psi_{-1}$ on the right.

Formally, determining whether the particle is in state $\Psi_{+1}$ or $\Psi_{-1}$ is a measurement because the two states are eigenstates of a self-adjoint operator. ${ }^{17}$ Merely satisfying this formal property might raise doubts as to whether this measurement is the sort of measurement that could actually be performed. The states $\Psi_{+1}$ or $\Psi_{-1}$ differ only in a relative internal phase factor. Is this a difference that measurement can reveal, or is it merely an unphysical gauge freedom?

We can put these doubts to rest. Recall that one instantiation of the two-chambered box is a separated singly ionized hydrogen molecule $\mathrm{H}_{2}{ }^{+}$. In this context, the two states have distinct physical meanings. For the ionized hydrogen molecule, the wavefunctions $\Psi_{\mathrm{L}}$ and $\Psi_{\mathrm{R}}$ correspond to $1 \mathrm{~s}$ electron orbitals of the hydrogen atom. For $\Psi_{+1}$, the two parts of the electron wavefunction are the same $1 s$ orbitals in phase. If the two hydrogen nuclei are allowed to approach, then molecular orbital theory, in the LCAO approximation, tells us that they form a stable lower energy $\sigma$ bond state. The bonding depends essentially on the zero phase difference (the +1 factor) between the two wavefunctions $\Psi_{\mathrm{L}}$ and $\Psi_{\mathrm{R}}$. Because of this zero phase difference, the two wavefunctions reinforce each other in the region of overlap between the two nuclei, increasing the wavefunction amplitude between the nuclei. $^{18}$

We find the opposite behavior for $\Psi_{-1}$. Then the two $1 s$ orbitals are $180^{\circ}$ out of phase (the -1 factor). As a result, where the two wavefunctions overlap, they will destructively interfere. Calculations show that this state has higher energy state. ${ }^{19}$ It is an unstable state in which the two nuclei repel (the antibonding $\sigma^{*}$ state). The two states are shown in Fig. 10 , where the magnitude of the wavefunction amplitude is indicated by shading.

The bonding and antibonding states enable a simple experimental procedure for determining whether the separated singly ionized hydrogen molecule $\mathrm{H}_{2}{ }^{+}$is in the bonding state

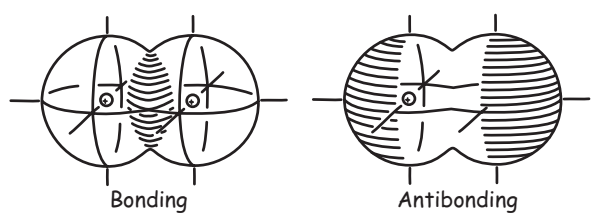

Fig. 10. Bonding and antibonding states of $\mathrm{H}_{2}^{+}$. 
$\Psi_{+1}$ or in the antibonding state $\Psi_{-1}$. The nuclei are allowed to move freely. If they are in the bonding state, they will attract and approach one another. If they are in the antibonding state, they will repel. ${ }^{20}$

It is not easy to discern how the state $\Psi_{\mathrm{ABC}}$ of Eq. (5) will behave under bonding/antibonding measurements, when those measurements are used to determine the position properties of a remote particle. We arrive at a more useful expression for the state if we make the following substitutions. The wavefunctions $\Psi_{\mathrm{C},+i}$ and $\Psi_{\mathrm{C},-i}$ are given in terms of $\Psi_{\mathrm{C}, \mathrm{L}}$ and $\Psi_{\mathrm{C}, \mathrm{R}}$ by means of the expressions

$$
\begin{aligned}
& \Psi_{\mathrm{C},+i}=(1 / \sqrt{2})\left(\Psi_{\mathrm{C}, \mathrm{L}}+i \Psi_{\mathrm{C}, \mathrm{R}}\right), \\
& \Psi_{\mathrm{C},-i}=(1 / \sqrt{2})\left(\Psi_{\mathrm{C}, \mathrm{L}}-i \Psi_{\mathrm{C}, \mathrm{R}}\right) .
\end{aligned}
$$

The wavefunctions $\Psi_{\mathrm{A},+i}, \Psi_{\mathrm{A},-i}, \Psi_{\mathrm{B},+i}$, and $\Psi_{\mathrm{B},-i}$ are given in terms of $\Psi_{\mathrm{A},+1}, \Psi_{\mathrm{A},-1}, \Psi_{\mathrm{B},+1}$, and $\Psi_{\mathrm{B},-1}$ by means of ${ }^{21}$

$$
\begin{aligned}
& \Psi_{\mathrm{A},+i}=((1+i) / 2)\left(\Psi_{\mathrm{A},+1}-i \Psi_{\mathrm{A},-1}\right), \\
& \Psi_{\mathrm{A},-i}=((1-i) / 2)\left(\Psi_{\mathrm{A},+1}+i \Psi_{\mathrm{A},-1}\right) \\
& \Psi_{\mathrm{B},+i}=((1+i) / 2)\left(\Psi_{\mathrm{B},+1}-i \Psi_{\mathrm{B},-1}\right), \\
& \Psi_{\mathrm{B},-i}=((1-i) / 2)\left(\Psi_{\mathrm{B},+1}+i \Psi_{\mathrm{B},-1}\right)
\end{aligned}
$$

Multiplying out the resulting terms and canceling where possible leads to the expression

$$
\begin{aligned}
\Psi_{\mathrm{ABC}}= & (i / 2)\left(\Psi_{\mathrm{A},+1} \Psi_{\mathrm{B},+1} \Psi_{\mathrm{C}, \mathrm{L}}-\Psi_{\mathrm{A},-1} \Psi_{\mathrm{B},-1} \Psi_{\mathrm{C}, \mathrm{L}}\right. \\
& \left.+\Psi_{\mathrm{A},+1} \Psi_{\mathrm{B},-1} \Psi_{\mathrm{C}, \mathrm{R}}+\Psi_{\mathrm{A},-1} \Psi_{\mathrm{B},+1} \Psi_{\mathrm{C}, \mathrm{R}}\right) .
\end{aligned}
$$

As before, we can see how measurements of bonding/ antibonding on the $\mathrm{A}$ and $\mathrm{B}$ particles enable us to predict the outcome of a position measurement on the $\mathrm{C}$ particle. If, for example, we measure both $\mathrm{A}$ and $\mathrm{B}$ particles to be in the bonding state, then the wavefunction of Eq. (13) has collapsed to

$$
\Psi_{\mathrm{ABC}}=\Psi_{\mathrm{A},+1} \Psi_{\mathrm{B},+1} \Psi_{\mathrm{C}, \mathrm{L}},
$$

and we are certain that a subsequent measurement on particle $\mathrm{C}$ will show an $\mathrm{L}$ position.

Because systems A, B, and C enter symmetrically in the state of Eq. (5), these results will hold no matter which two are the particles on which bonding/antibonding measurements are performed. Hence, from Eq. (13), we can read off the following rule, similar to that found for position measurements:

$$
\text { Rule: Same — L, Different — R. }
$$

If we measure two particles to have the same bonding state, then the remaining particle will return position $\mathrm{L}$ on measurement. If we measure two particles to have different bonding states, then the remaining particle will return position R on measurement. Here, same just means both bonding or both antibonding, and different means one bonding and one antibonding.

The point is that the role of $\mathrm{L}$ and $\mathrm{R}$ in the rule for bonding measurements is reversed in comparison to the rule associated with position measurements. This reversal will yield the contradiction.

\section{Applying the EPR reality criterion}

We proceed as before by assembling the outcomes of all possible bonding measurements on pairs of particles. These bonding measurements must cohere with one another. ${ }^{22}$ Hence, there are only two cases for the various pairs of bonding measurements on the three particles: (a) All three pairs have the same bonding states. (b) One pair has the same bonding state and the remaining two pairs have different bonding states. The position properties of the remote unmeasured particles must all conform to the rule in Eq. (15). Applying it to case (a) tells us that the three position properties must all be L. Applying the rule to case (b) tells us that one position property is $\mathrm{L}$ and that the other two must be $\mathrm{R}$. That is, there are only four possible distributions of the position properties: RRL, RLR, LRR, or LLL. We see immediately that these four distributions of position properties contradict the distribution of position properties in Eq. (9) inferred from position measurements.

\section{CONCLUSION}

We have arrived at a contradiction. Position measurements on the three entangled particles of quantum state [Eq. (5)] lead to the conclusion that the particles must possess position properties that contradict those inferred from bonding/ antibonding measurements.

Two principal assumptions were made in the arguments that generated this contradiction. One was that the empirical predictions of quantum theory are reliable. The other was the EPR reality criterion, which, in turn, depends on the assumptions of separability and locality. One of these assumptions must be given up. The continuing empirical success of quantum theory has led to a consensus that it is the second assumption, the EPR reality criterion, which is to be discarded.

\section{ACKNOWLEDGMENTS}

The author is grateful to Julia Bursten, Simon Garcia, Eugen Schwarz, and Michael Weisberg for helpful guidance on chemical bonding. The author also thanks Diana Buchwald, Tony Duncan, Wayne Myrvold, David Snoke, Travis Norsen, Giovanni Valente, and Allan Walstad for their advice and assistance.

\footnotetext{
${ }^{1}$ A. Einstein, B. Podolsky, and N. Rosen, "Can quantum-mechanical description of physical reality be considered complete?," Phys. Rev. 47, 777-780 (1935); A. Einstein, "Physics and reality," J. Franklin Inst. 221, 349-382 (1936); A. Einstein, "Autobiographical notes" in Albert Einstein: Philosopher-Scientist, 2nd ed., edited by P. A. Schilpp (Tudor, New York, 1951), pp. 1-94; "Quanten-mechanik und wirklichkeit," Dialectica 2, 320-324 (1948).

${ }^{2}$ J. S. Bell, "On the Einstein-Podolsky-Rosen paradox," Phys. 1, 195-200 (1964); reprinted in Speakable and Unspeakable in Quantum Physics (Cambridge U. P., Cambridge, 1987), Chap. 2, pp. 14-15.

${ }^{3}$ Although none of Einstein's published versions of his attempts to show incompleteness employed spin degrees of freedom, Tilman Sauer recently found an unpublished Einstein manuscript of late 1954 or early 1955 in which the analysis is developed in terms of spin. T. Sauer, "An Einstein manuscript on the EPR paradox for spin observables," Stud. Hist. Philos. Mod. Phys. 38, 879-887 (2007).

${ }^{4}$ H. J. Bernstein, "Simple version of the Greenberger-Horne-Zeilinger (GHZ) argument against local realism," Found. Phys. 29, 521-525 (1999).

${ }^{5}$ Einstein Archive, Document 22-047. Portions of this letter have been reproduced in German and in English translation in the work by Arthur Fine and Don Howard. My translation is a variation of theirs. A. Fine,
} 
The Shaky Game: Einstein, Realism and the Quantum Theory (University of Chicago Press, Chicago, 1986), p. 69; D. Howard, "Einstein on locality and separability," Stud. Hist. Philos. Sci. 16, 171-201 (1985).

${ }^{6}$ Translation from Howard, Ref. 5.

${ }^{7}$ Einstein's emphasis, Ref. 6. T. Norsen, "Einstein's boxes," Am. J. Phys. 73, 164-176 (2005), also developed the example of Einstein's boxes. However, he considered only one quantity, position, in contrast to the noncommuting quantities of the present analysis $\mathrm{He}$ arrived at weaker results, as pointed out by A. Shimony, "Comment on Norsen's defense of Einstein's 'box argument'," ibid. 73, 177-178 (2005).

${ }^{8}$ For an extended treatment of the solution of Schrödinger's equation for square potentials, see D. Bohm, Quantum Theory (Prentice-Hall, Englewood Cliffs, NJ, 1951); Quantum Theory (Dover, Mineola, NY, 1989), Chap. 11.

${ }^{9}$ C. J. Ballhausen and H. B. Gray, Molecular Orbital Theory (Benjamin, New York, 1965), Chap. 2.1.

${ }^{10}$ P. Atkins, Physical Chemistry (Freeman, New York, 1994), Fig. 14.18.

${ }^{11}$ This allowance of counterfactual measurement appears in the EPR analysis in the consideration of measurements on the same system of observables corresponding to noncommuting operators, such as, in their example, the position and the momentum of one of the particles. Both measurements are considered, but at most one can be carried out.

${ }^{12}$ Bohr's celebrated response to the EPR paper depends on insisting that, were different measurements performed, the system would somehow be different. As a result, considering different measurements counterfactually on the first system no longer enables us to extend the list of properties we infer as possessed by the remote system. For a mature version, see N. Bohr, "Discussions with Einstein on epistemological problems in atomic physics," in Albert Einstein-Philosopher Scientist, 2nd ed., edited by P. A. Schilpp (Tudor, New York, 1951), pp. 201-241. Assessments of Bohr's response to the EPR paper vary widely. In my view, Bohr's response fails because it depends on an extreme form of empiricism that conflates what a system is with how we find out about it. Standard quantum theory does not make that conflation. It provides clear statements as to what would happen were, counterfactually, different measurements to be performed on one and the same quantum system.

${ }^{13}$ D. Greenberger, M. Horne, and A. Zeilinger, "Going beyond Bell's theorem," in Bell's Theorem, Quantum Theory, and Conceptions of the Universe, edited by M. Kafatos (Kluwer, Dordrecht, 1989), pp. 73-76. See also R. Clifton, M. L. G. Redhead, and J. Butterfield, "Generalization of the Greenberger-Horne-Zeilinger algebraic proof of nonlocality," Found. Phys. 21, 149-184 (1991).

${ }^{14}$ D. Mermin, "What's wrong with these elements of reality?," Phys. Today 43(6), 9-11 (1990).
${ }^{15}$ The substitution depends on the fact that the two states $\Psi_{\mathrm{L}}$ and $\Psi_{\mathrm{R}}$ are orthogonal because they are nonzero only in spatially disjoint regions. Hence, they span a two-dimensional Hilbert space, which can be substituted for the traditional spin-1/2 spaces employed in GHZ constructions. One mapping associates states $\Psi_{\mathrm{L}}$ and $\Psi_{\mathrm{R}}$ with z-spin up and z-spin down; states $\Psi_{i}$ and $\Psi_{-i}$ of Eq. (1) with y-spin up and y-spin down; and states $\Psi_{1}$ and $\Psi_{-1}$ of Eq. (1) with $\mathrm{x}$-spin up and $\mathrm{X}$-spin down. This mapping enables a conversion between a GHZ construction in terms of spin properties and the construction used in this paper.

${ }^{16}$ The same rule applies to all cases because of the manifest symmetry of the state of Eq. (5) in the three particles: A, B, and C.

${ }^{17}$ As noted in Ref. 15, the two orthogonal states, $\Psi_{\mathrm{L}}$ and $\Psi_{\mathrm{R}}$, span a two-dimensional Hilbert space. Because $\Psi_{+1}$ or $\Psi_{-1}$ are also orthogonal, the self-adjoint operator acting on this space with eigenvectors $\left|\Psi_{+1}\right\rangle$ or $\left|\Psi_{-1}\right\rangle$ is just $\left|\Psi_{+1}\right\rangle\left\langle\Psi_{+1}|-| \Psi_{-1}\right\rangle\left\langle\Psi_{-1}\right|$.

${ }^{18}$ See Refs. 9 and 10, Sec. 14.4. For a crude heuristic picture, imagine that this reinforcement means the single electron tends to inhabit the space between the nuclei more so that its attraction for the nuclei pulls them together. Alternatively, the closer packing of the three particles (two nuclei and one electron) leads to a lower energy state.

${ }^{19}$ For a crude heuristic picture, this interference means that the three particles are more spread out and thus have a higher energy state. We might also imagine the electron more on the outside of the two nuclei so that its attraction for the nuclei will separate them.

${ }^{20}$ At the level of the LCAO approximation, the energies of the states $\Psi_{+i}$, $\Psi_{-i}, \Psi_{\mathrm{L}}$, and $\Psi_{\mathrm{R}}$ are independent of the internuclear distance, so none of these states form bonding or antibonding interactions. Hence, testing for attraction and repulsion tests specifically for the states $\Psi_{+1}$ and $\Psi_{-1}$.

${ }^{21}$ To see that these are the correct expressions for $\Psi_{\mathrm{A},+i}$, etc., substitute in them for $\Psi_{\mathrm{A},+1}$, etc., using Eq. (10), and recover the expressions in Eq. (4) for $\Psi_{\mathrm{A},+i}$, etc.

${ }^{22}$ This assertion depends on the further assumption that the bonding state of a particle is a property possessed by it, akin to its possession of a position property. This further assumption can be established by an EPR style analysis akin to that used to establish the reality of the position properties. Assume that we measure the bonding state of particle A and the position of particle C. Equation (13) allows us to predict with certainty which bonding state the B particle will show under measurement. If, for example the A particle is bonding and the $\mathrm{C}$ particle is $\mathrm{L}$, then $\Psi_{\mathrm{ABC}}$ collapses to the first term of Eq. (13), and we can predict with certainty that a bonding measurement on the $\mathrm{B}$ particle will show it to be bonding. Analogous analysis with other pairs leads to the conclusion that each particle possesses the property of a definite bonding state.

\section{MAKE YOUR ONLINE MANUSCRIPTS COME ALIVE}

A picture is worth a thousand words. Film or animation can be worth much more. If you submit a manuscript which includes an experiment or computer simulation, why not make a film clip of the experiment or an animation of the simulation, and place it on the Supplementary Material server. Your online manuscript will have a direct link to your supplementary material.

See http://www.kzoo.edu/ajp/EPAPS.html for more information. 